\title{
Solve Partial Differential Equations using the Banach Contraction Method and Improve Results Using the Trapezoidal Rule
}

\author{
Ahmed E. Ghitheeth \\ ahmed_entesar84@uomosul.edu.iq \\ Department of Mathematics \\ College of Computer Science and Mathematics \\ University of Mosul, Mosul, Iraq
}

\author{
Hiba Sh. Mahmood \\ hiba.sh@uomosul.edu.iq \\ Department of Mathematics \\ College of Education for Pure Science \\ University of Mosul, Mosul, Iraq
}

Received on: 04/03/2021

Accepted on: 04/04/2021

\begin{abstract}
In this paper, the Banach contraction method (BCM) was hybridized with the base of the trapezoid (TR) to solve non-linear partial differential equations. The results obtained from (BCM) were improved by (TR). The results obtained from (BCM -TR) proved the efficiency of this method compared to the default method (BCM). This was done by calculating the mean square error (MSE) and estimating the absolute maximum error (MAE).
\end{abstract}

Keywords: Banach contraction Method, Trapezoidal Rule, Partial differential equations.

$$
\begin{aligned}
& \text { حل المعادلات التفاضلية الجزئية باستخدام طريقة باناخ للتقلص } \\
& \text { وتحسين النتائج باستخدام قاعدة شبه منحرف } \\
& \text { هبة شكر محمود } \\
& \text { أحد انتصار غثيث } \\
& \text { قسم الرياضيات، كلية التربية للعلوم الصرفة } \\
& \text { جامعة الموصل، الموصل، العراق } \\
& \text { قسم الرياضيات، كلية علوم الحاسوب والرياضيات } \\
& \text { جامعة الموصل، الموصل، العرق }
\end{aligned}
$$

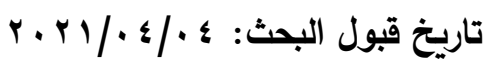

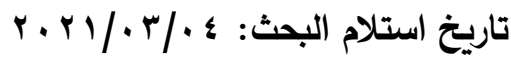

\section{الملغص}

\section{Introduction}

Partial differential equations have many important applications in various disciplines (science and engineering) and others. There are many ways to solve this type of equations, numerical or analytical methods, such as the analytical Adomian method [1], the variable iteration method [2], the residual power chain method [3] and there are many other methods, and these methods have proven high efficiency in solving These equations and reach good results. 
The fixed point theory is called Banach (the principle of contraction maps) and it is one of the important and fundamental methods in the metric area theory because it means the presence and uniqueness between the fixed points of some original maps of the metric area, and the name of this theory was derived from Stefan Banach [18921945] and was first mentioned in 1922 [4]. The BCM method is a development of the Picard method and it is easy to implement, which distinguishes it from other iterative method. The BCM method is applied in solving some important problems in engineering and physics

Numerical analysis was used in solving differential equations, and in this paper, the trapezoid rule was used, which is one of the ancient and well-known numerical methods for approximating the specific integral, representing the mean left and right Riemann sum as the best approximation by dividing the integration period, is more precisely approximated by dividing the integral into multiple periods, after which this rule is applied to each period, and then we summation the results [5].

We will solve the partial equation whose general form is:

$\mathcal{L}_{t} z(x, t)+\mathcal{L}_{x} z(x, t)+\operatorname{Rz}(x, t)+\mathrm{Nz}(x, t)=s(x, t)$

with the initial conditions

$z(x, 0)=\mathrm{H}(x, t), z_{t}(x, 0)=\rho(x, t)$

Where as $\mathcal{L}_{t=\frac{d z}{d t}}$ or $\mathcal{L}_{t=\frac{d^{2} z}{d t^{2}}}$ is a highest rank difference in $t, \mathcal{L}_{x}$ is a highest rank difference in $x, \mathrm{R}$ is the differential factor and consists of derivatives of a lesser order, $\mathrm{N}$ is a nonlinear analytical term and $s(x, t)$ is the defined heterogeneous term [6].

The research is organized as follows: definitions and theorems in section 2, basic rules for $(\mathrm{BCM})$ are mentioned in section 3, the proposed technique of (BCM-TR) is mentioned in section 4, applications and results are mentioned in section 5, conclusions are mentioned in section 6 .

\section{Basic Concepts}

Some fundamental meanings and concepts relevant to the research topic will be discussed in this section.

2.1 Let $(B, d)$ It must be a metric space then a mapping $\mathrm{T}: B \rightarrow B$ is said to be Lipschitz if a positive real number exists $\geq 0$, as a result $\forall b, h \in B$ implies $d\left(T_{b}, T_{h}\right) \leq k d(b, h)$

Furthermore if $k<1$, then $\mathrm{T}$ is called a contraction mapping on $B$ [7].

\subsection{Theorem of Banach Fixed Points:}

Let's (B,d) be such a complete metric space, where $B \neq \emptyset$ also T: $B \rightarrow B$ be a contraction on $B$, after this T does have a unique fixed-point $b^{*} \in B$ if $\mathrm{T}\left(b^{*}\right)=b^{*}$

\section{Theorem 2.3: [8]}

Let $(B, d)$ It must be a complete metric space $\&$ let T: $B \rightarrow B$ be a mapping such that $\mathrm{T}^{k}$ is a contraction mapping of $B$ for most any real positive number $k$. Then $\mathrm{T}$ has a unique fixed point in $B$.

\subsection{Banach Contraction Principle [9].}

let $(B, d)$ is complete metric space and $\mathrm{T}: B \rightarrow B$ is a contraction mapping with Lipchitz constant $k$, that T has a unique fixed point $b_{0}$ in $B$ as well as each $b \in B$, We've 
got $\lim _{n \rightarrow \infty} \mathrm{T}^{n}(b)=b_{0}$ and Furthermore, for each $b \in B$, We've got $d\left(\mathrm{~T}^{n}(b), b_{0}\right) \leq$ $\frac{\kappa^{n}}{1-\kappa} d(\mathrm{~T}(b), b)$

\subsection{The Maximum Absolute Error (MAE) [10]:}

It is defined as the greatest absolute value of the difference between an exact solution and an approximate solution as follows:

$\left\|z_{\text {Exact }}(t)-\Phi_{q}(t)\right\|_{\infty}=\max _{a \leq x \leq b}\left\{\left|z_{\text {Exact }}(t)-\Phi_{q}(t)\right|\right\}$

\subsection{Mean Square Error (MSE):}

It mean square of error is calculated as the sum of the square of the difference between the exact solution $E t\left(t_{\tau}\right)$ and the approximate solutions $\Phi\left(t_{\tau}\right)$ divided by the number of points used $\mathrm{M}$ and as in the following formula:

$M S E=\frac{\sum_{\tau=1}^{\mathrm{M}}\left(E t\left(t_{\tau}\right)-\Phi\left(t_{\tau}\right)\right)^{2}}{\mathrm{M}}$

Where $\vec{t}_{\tau}$ are vectors since $\tau=1,2, \ldots$

\section{Banach Contraction Method (BCM)}

Recognize the functional nonlinear equation.

$\mathrm{z}(\bar{t})=s(\bar{t})+N(\mathrm{z}(\bar{t}))$

We define the successive approximations as follows

$z_{0}=s$

$\mathrm{z}_{1}=\mathrm{z}_{0}+\mathrm{N}\left(\mathrm{z}_{0}\right)$

$\mathrm{z}_{2}=\mathrm{z}_{0}+\mathrm{N}\left(\mathrm{z}_{1}\right)$

$\vdots$

$\mathrm{z}_{\mathrm{I}}=\mathrm{z}_{0}+\mathrm{N}\left(\mathrm{z}_{\mathrm{I}-1}\right), \quad \mathrm{I}=1,2, \cdots$,

the solution of (3) We get it by

$\mathrm{z}=\lim _{\mathrm{I} \rightarrow \infty} \mathrm{z}_{\mathrm{I}}$

\section{The Proposed Technique BCM-TR} get

By adding the operator $\mathcal{L}_{t}{ }^{-1}$ which is the inverse of $\mathcal{L}_{t}$ to equation (1) and (2), we $z(x, t)=f(x, t)+\mathcal{L}_{t}^{-1}\left(\mathcal{L}_{x} z-\mathrm{Rz}-\mathrm{Nz}\right)$

We obtain the following successive approximations:

$z_{0}=f(x, t)$

$z_{1}=z_{0}+\mathcal{L}_{t}^{-1}\left(\mathcal{L}_{x} z_{0}-\mathrm{R} z_{0}-\mathrm{N} z_{0}\right)$

$z_{2}=z_{0}+\mathcal{L}_{t}^{-1}\left(\mathcal{L}_{x} z_{1}-\mathrm{R} z_{1}-\mathrm{N} z_{1}\right)$

$\vdots$

$z_{\mathrm{I}}=z_{0}+\mathcal{L}_{t}^{-1}\left(\mathcal{L}_{x} z_{z_{\mathrm{I}-1}}-\mathrm{R} z_{z_{\mathrm{I}-1}}-\mathrm{N} z_{z_{\mathrm{I}-1}}\right), \quad \mathrm{I}=1,2, \cdots$

Now, Banach's contraction method has been linked to the trapezoidal base by using the series (9) when $I=3$ and considering it an elementary solution and then solving the example again with the base of a trapezoid and obtaining numerical results.

\section{Illustrative Examples}

In this section, three different models of non-linear homogeneous and heterogeneous partial differential equations were solved using the method (BCM-TR), and we obtain numerical results by using Maple. 


\section{Example 1 [11]:}

Let us use the following Burgers' nonlinear partial problem of the first order:

$z_{t}(x, t)+z(x, t) z_{x}(x, t)=z_{x x}(x, t)$

with condition $z(x, 0)=2 x$

The exact solution is $z_{\text {Exact }}(x, t)=\frac{2 x}{1+2 t}$.

Following the technique mentioned in Part 4, the following iterations are done:

$z_{0}=2 x$

$z_{1}=2 x-4 x t$

$z_{2}=2 x-4 x t+8 x t^{2}-\frac{16}{3} x t^{3}$

$z_{3}=2 x-4 x t+8 x t^{2}-16 x t^{3}+\frac{64}{3} x t^{4}-\frac{64}{3} x t^{5}+\frac{128}{9} x t^{6}-\frac{256}{63} x t^{7}$

Finally, take the series (11) and solve it using the trapezoid rule.

Table 1: Compare both (BCM) and (BCM_TR) when $t=0.5$ for Example. 1

\begin{tabular}{ccc}
\hline Error Scale & (BCM) & (BCM_TR) \\
\hline MAE & 0.14285 & 0.00831 \\
\hline MSE & 0.00714 & $2.41847 \mathrm{E}-05$ \\
\hline
\end{tabular}

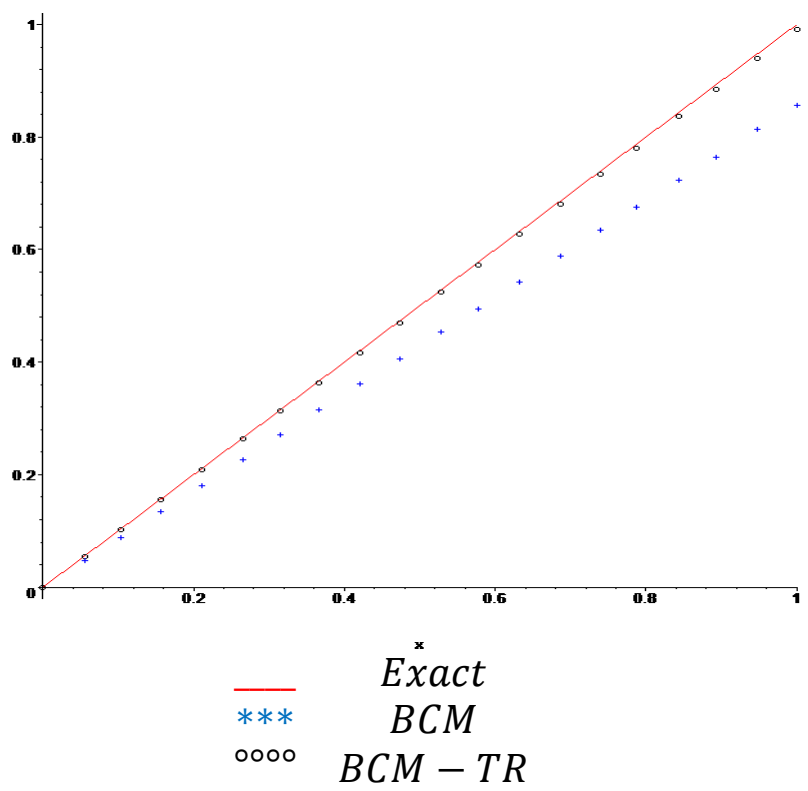

Example 2[12]:

Let us have the non-linear partial advection problem of the following first order: $z_{t}(x, t)+z(x, t) z_{x}(x, t)=0$

where condition $z(x, 0)=-x$

The exact solution is $z_{\text {Exact }}(x, t)=\frac{x}{t-1}$.

Following the technique mentioned in Part 4, the following iterations are done: $z_{0}=-x$

$z_{1}=-x-x t$

$z_{2}=-x-x t-x t^{2}-\frac{1}{3} x t^{3}$

$z_{3}=-x-x t-x t^{2}-x t^{3}-\frac{2}{3} x t^{4}-\frac{1}{3} x t^{5}-\frac{1}{9} x t^{6}-\frac{1}{63} x t^{7}$

Finally, take the series (13) and solve it using the trapezoid rule. 
Table 2: Compare both (BCM) and (BCM_TR) when $t=0.6$ for Example. 2

\begin{tabular}{ccc}
\hline Error Scale & $(\mathbf{B C M})$ & (BCM_TR) \\
\hline MAE & 0.20605 & 0.01094 \\
\hline MSE & 0.01486 & $4.19525 \mathrm{E}-05$ \\
\hline
\end{tabular}

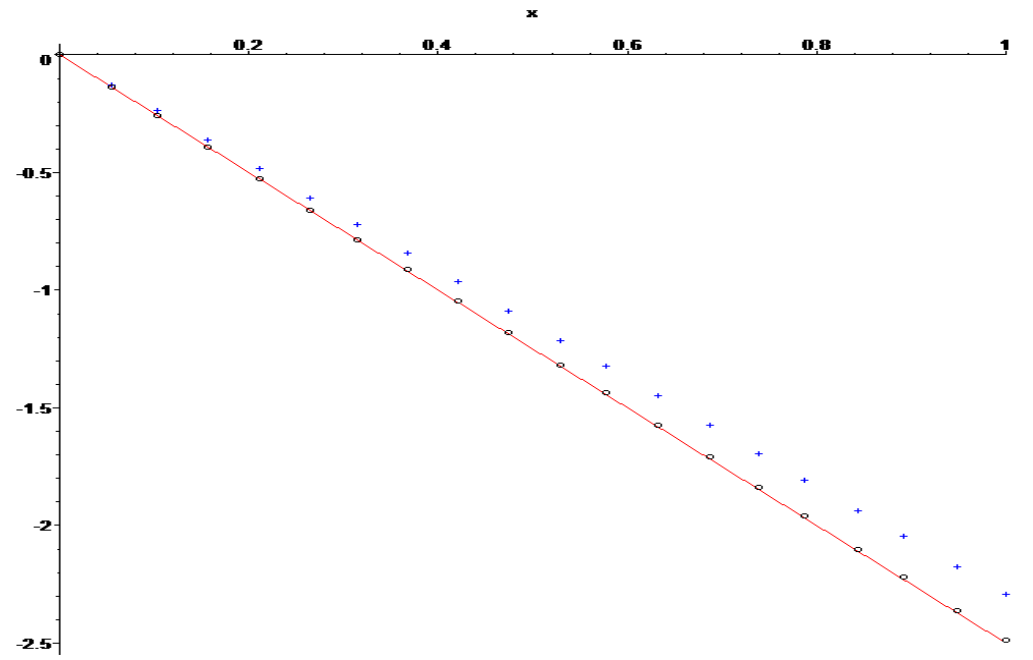

$$
\begin{array}{cc} 
& \text { Exact } \\
\hline * * * & B C M \\
\text { ०००० } & B C M-T R
\end{array}
$$

\section{Example 3 [13]:}

Let us use the following Second -order non-linear partial problem:

$z_{t t}(x, t)-z(x, t) z_{x x}(x, t)=1-0.5 t^{2}+0.5 x^{2}$

with conditions

$z(x, 0)=0.5 x^{2}$

$z_{t}(x, 0)=0$

The exact solution is $z_{\text {Exact }}(x, t)=0.5 t^{2}+0.5 x^{2}$.

Following the technique mentioned in Part 4, the following iterations are done:

$z_{0}=0.5 x^{2}+0.04166 t^{4}-0.5\left(1+0.5 x^{2}\right) t^{2}$

$z_{1}=0.5 x^{2}-0.04166 x^{2} t^{4}-0.5\left(1+0.5 x^{2}\right) t^{2}+0.25 x^{2} t^{2}+0.310 e^{-30} t^{4}$ $+0.009722 t^{6}-0.00037202 t^{8}+0.0041666 x^{2} t^{6}$

$z_{2}=0.5 x^{2}-0.5\left(1+0.5 x^{2}\right) t^{2}+0.12917 e^{-10} t^{16}+0.61549 e^{-6} t^{14}+$

$0.19078 e^{-6} x^{2} t^{14}-0.61377 e^{-5} t^{12}-0.52609 e^{-5} x^{2} t^{12}-0.50429 e^{-5} t^{10}+$ $0.39580 e^{-4} x^{2} t^{16}+\cdots$.

$$
\begin{aligned}
z_{3}=0.5 x^{2}- & 0.5\left(1+0.5 x^{2}\right) t^{2}+0.25 x^{2} t^{2}+0.17857 e^{-33} t^{8}+0.26979 e^{-7} x^{2} t^{14} \\
& +0.58454 e^{-6} x^{2} t^{12}+0.33061 e^{-5} x^{2} t^{10}-0.99206 e^{-4} x^{2} t^{8} \\
& +0.32430 e^{-8} t^{16}-0.24570 e^{-6} t^{14}+\cdots
\end{aligned}
$$

Finally, take the series (15) and solve it using the trapezoid rule.

Table 3: Compare both (BCM) and (BCM_TR) when $t=0.25$ for Example.3

\begin{tabular}{ccc}
\hline Error Scale & $(\mathbf{B C M})$ & (BCM_TR) \\
\hline MAE & 0.062500 & 0.014574 \\
\hline MSE & 0.003906 & $7.64184 \mathrm{E}-05$ \\
\hline
\end{tabular}




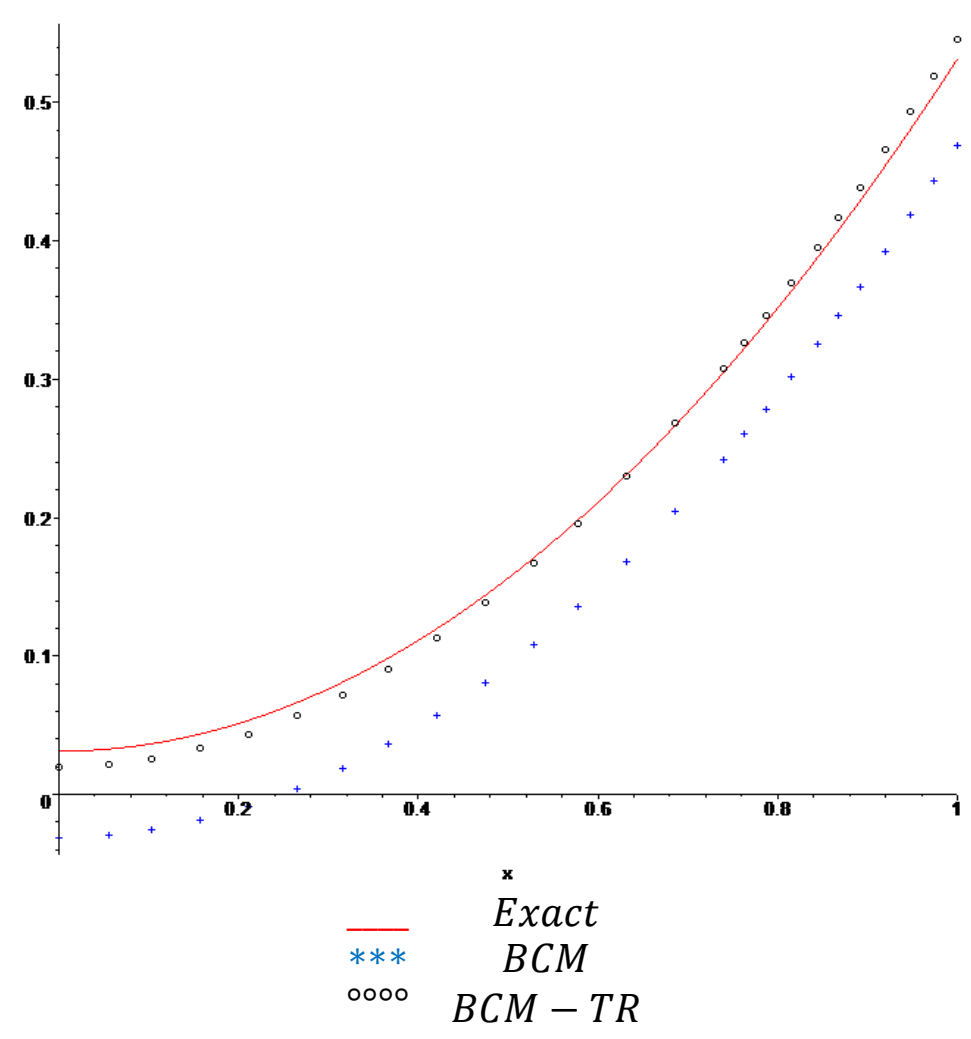

\section{Conclusions}

In this paper, first and second-order partial differential equations are solved using (BCM-TR) method. We obtained good numerical results compared to the hypothetical method by computing the mean square error (MSE) and maximum absolute error (MAE) as shown in Tables $(1,2,3)$. The drawing also shows the efficiency and accuracy of this method. 


\section{REFERENCES}

[1] G. Adomian, "Nonlinear dissipative wave equations," Appl. Math. Lett., vol. 11, no. 3, pp. 125-126, 1998.

[2] M. Inokuti, H. Sekine, and T. Mura, "General use of the Lagrange multiplier in nonlinear mathematical physics," Var. method Mech. solids, vol. 33, no. 5, pp. 156-162, 1978.

[3] M. İnç, Z. S. Korpinar, M. Al Qurashi, and D. Baleanu, "A new method for approximate solutions of some nonlinear equations: Residual power series method," Adv. Mech. Eng., vol. 8, no. 4, p. 1687814016644580, 2016.

[4] I. Altun and H. Simsek, "Some fixed point theorems on ordered metric spaces and application," Fixed Point Theory Appl., vol. 2010, pp. 1-17, 2010.

[5] Q. I. Rahman and G. Schmeisser, "Characterization of the speed of convergence of the trapezoidal rule," Numer. Math., vol. 57, no. 1, pp. 123-138, 1990.

[6] M. Hussain and M. Khan, "Modified Laplace decomposition method," Appl. Math. Scie, vol. 4, pp. 1769-1783, 2010.

[7] E. Kreyszig, Introductory functional analysis with applications, vol. 1. wiley New York, 1978.

[8] M. C. Joshi and R. K. Bose, Some topics in nonlinear functional analysis. John Wiley \& Sons, 1985.

[9] V. Daftardar-Gejji and S. Bhalekar, "Solving nonlinear functional equation using Banach contraction principle," Far East J. Appl. Math., vol. 34, no. 3, pp. 303314, 2009.

[10] W. Al-Hayani, L. Alzubaidy, and A. Entesar, "Solutions of singular IVP's of Lane-Emden type by homotopy analysis method with genetic algorithm," Appl. Math. Inf. Sci, vol. 11, no. 2, pp. 407-416, 2017.

[11] M. M. Azeez, Semi-Analytical Iterative Methods for Non-Linear Differential Equations. 2017.

[12] J. PATRA, "Some Problems on Variational Iteration Method," no. May, pp. 1$35,2015$.

[13] A. Khatib, "Differential Transform Method for Some Delay Differential Equations," Appl. Math., vol. 06, no. 03, pp. 585-593, 2015. 